\title{
Aspartate Aminotransferase to Creatine Kinase Ratio Measurement
}

National Cancer Institute

\section{Source}

National Cancer Institute. Aspartate Aminotransferase to Creatine Kinase Ratio

Measurement. NCI Thesaurus. Code C158225.

The determination of the ratio of aspartate aminotransferase compared to creatine kinase present in a sample. The measurement may be expressed as a ratio or percentage. 